\title{
ANOTHER CHARACTERISATION OF PFAFFIAN BIPARTITE GRAPHS
}

\author{
CHARLES H. C. LITTLE
}

(Received 23 April 1982; revised 29 November 1983)

Communicated by W. D. Wallis

\begin{abstract}
It has been known for over twenty years that every planar graph is Pfaffian. Recently a characterisation of planar graphs in terms of strict maximal odd rings has been discovered. This paper attempts to elucidate the connection between the Pfaffian property and planarity by characterising Pfaffian bipartite graphs in terms of maximal odd rings.
\end{abstract}

1980 Mathematics subject classification (Amer. Math. Soc.): 05 C 70.

\section{Introduction}

All graphs considered in this paper are finite. Let $G^{*}$ be a directed graph with an even number of vertices, and let $F$ be the set $\left\{f_{1}, \ldots, f_{k}\right\}$ of its 1 -factors. For all $i$ write $f_{i}=\left\{\left(u_{i 1}, w_{i 1}\right),\left(u_{i 2}, w_{i 2}\right), \ldots,\left(u_{i n}, w_{i n}\right)\right\}$ where $n=\frac{1}{2}\left|V\left(G^{*}\right)\right|$ and, for all $j$, $\left(u_{i j}, w_{i j}\right)$ denotes an edge directed from vertex $u_{i j}$ to vertex $w_{i j}$. Associate with $f_{i}$ a plus sign if $u_{i 1} w_{i 1} u_{i 2} w_{i 2} \cdots u_{i n} w_{i n}$ is an even permutation of $u_{11} w_{11} u_{12} w_{12} \cdots$ $u_{1 n} w_{1 n}$, and a minus sign otherwise. If $G$ is an undirected graph, we say that $G$ is a Pfaffian graph if there exists a directed graph $G^{*}$ with vertex set $V(G)$ and edge set $E(G)$ such that all the 1-factors of $G^{*}$ have the same sign.

The idea of affixing signs to the 1-factors of a directed graph in this manner is due to Kasteleyn [2], who showed that every planar graph is Pfaffian. Pfaffian bipartite graphs have been characterised in [3]. In order to describe this characterisation, we need some more notation. Let $G$ be a bipartite graph with

(C) 1985 Australian Mathematical Society $0263-6115 / 85 \$ A 2.00+0.00$ 
bipartition $\left\{V, V^{\prime}\right\}$. (In other words, every edge of $E(G)$ joins a vertex of $V$ to one of $V^{\prime}$.) Let $f$ be a 1-factor of $G$. Then $G f$ denotes the directed graph obtained from $G$ by orienting each edge of $f$ away from the end in $V$ and each edge of $E(G)-f$ toward the end in $V$. Furthermore, let $H$ be the directed graph of Figure 1. Then the following theorem is the characterisation given in [3].

THEOREM 1. A bipartite graph $G$ is non-Pfaffian if and only if there exists a 1-factor $f$ of $G$ such that some subgraph of $G$ fis isomorphic to a subdivision of $H$.

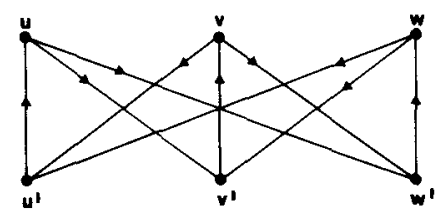

Figure 1

The connection between planarity and the Pfaffian property described above is perhaps rather unexpected. In this paper, we attempt to elucidate that connection by appealing to a recent characterisation of planarity. We turn now to a description of that characterisation.

If $S$ is a set of circuits of $G$, then the circuits in $S$ are consistently orientable if $G$ can be oriented so that they are all directed circuits. A ring of circuits in $G$ is a set $S$ of consistently orientable circuits such that

(a) $|S| \geqslant 3$,

(b) there is a cyclic ordering $\left(C_{0}, C_{1}, \ldots, C_{n-1}, C_{0}\right)$ of the $n$ circuits in $S$ such that $E\left(C_{i}\right) \cap E\left(C_{j}\right) \neq \varnothing$ if and only if $j=i, j \equiv i-1(\bmod n)$ or $j \equiv i+1$ $(\bmod n)$, and

(c) no edge of $G$ belongs to more than two circuits of $S$.

A ring $S$ is said to be odd if $|S|$ is odd. A ring $\left\{C_{0}, \ldots, C_{n-1}\right\}$ is said to be maximum if there does not exist a ring $\left\{C_{0}^{\prime}, \ldots, C_{m-1}^{\prime}\right\}$ for which $m>n$ and

$$
\bigcup_{j=0}^{m-1} E\left(C_{j}^{\prime}\right) \subseteq \bigcup_{j=0}^{n-1} E\left(C_{j}\right) \text {. }
$$

The ring $\left\{C_{0}, \ldots, C_{n-1}\right\}$ is strict if $\left|V\left(C_{i}\right) \cap V\left(C_{j}\right)\right| \leqslant 1$ whenever $E\left(C_{i}\right) \cap E\left(C_{j}\right)$ $=\varnothing$.

The following characterisation of planar graphs has been proved by Chernyak [1]. 
THEOREM 2. A graph is non-planar if and only if it contains a maximum strict odd ring.

Chernyak's proof depends on work by Holton and Little that has not yet been published. See [4] for a self-contained proof for cubic graphs.

In the subsequent sections, we characterise Pfaffian bipartite graphs in terms of rings. The reader is referred to [4] for definitions and notation not explained here.

\section{Some preliminary results}

Our aim is to characterise Pfaffian bipartite graphs. We shall re-interpret the problem as one about cubic graphs, and shall solve the resulting problem concerning cubic graphs.

Throughout the following, $G$ is a bipartite graph and $f$ is a 1 -factor of $G$. We denote by $G^{*} f$ the cubic directed graph constructed from $G f$ in the following way. Delete every vertex of valency 1 (and the edge incident on it). Let $u$ be any vertex of valency 2 , and let $u_{1}$ and $u_{2}$ be the vertices adjacent to $u$. Since no vertex of $G f$ of valency greater than 1 is a source or sink, we may assume that $\left(u_{1}, u\right) \in E(G f)$ and $\left(u, u_{2}\right) \in E(G f)$. Then replace $u,\left(u_{1}, u\right)$ and $\left(u, u_{2}\right)$ by the single edge $\left(u_{1}, u_{2}\right)$. In this way, we remove all vertices of valency 2 . Finally, let $v$ be a vertex of valency $j>3$. Let $v_{1}, \ldots, v_{j}$ be the vertices adjacent to $v$, and let $\left(v_{1}, v\right)$ be the unique edge of $G f$ directed toward $v$. Replace $\left(v_{1}, v\right),\left(v, v_{2}\right),\left(v, v_{3}\right), \ldots,\left(v, v_{j}\right)$ and $v$ by the edges $\left(v_{1}, w_{2}\right),\left(w_{2}, v_{2}\right),\left(w_{2}, w_{3}\right),\left(w_{3}, v_{3}\right),\left(w_{3}, w_{4}\right),\left(w_{4}, v_{4}\right)$, $\left(w_{4}, w_{5}\right), \ldots,\left(w_{j-2}, v_{j-2}\right),\left(w_{j-2}, w_{j-1}\right),\left(w_{j-1}, v_{j-1}\right),\left(w_{j-1}, v_{j}\right)$, where $w_{2}$, $w_{3}, \ldots, w_{j-1}$ are new vertices not in $V(G f)$.

We use the corresponding procedure if there is a unique edge of $G f$ directed away from $v$. In this way all vertices of degree greater than 3 are elminated.

The following two lemmas are easily proved.

LEMMA 1. $G^{*} f$ contains a subdivision of $H$ if and only if $G f$ does.

LEMMA 2. $G^{*} f$ contains a maximum odd ring if and only if $G f$ does.

We conclude this section with a lemma that is proved in [3].

LEMMA 3. Let $f$ and $f^{\prime}$ be distinct 1-factors of a bipartite graph $G$. Then $G f^{\prime}$ is obtained from $G f$ by reversing the orientation of every edge of $f \oplus f^{\prime}$. 
Here $f \oplus f^{\prime}$ denotes the symmetric difference of $f$ and $f^{\prime}$. Note that it is the union of vertex-disjoint circuits.

\section{The main theorem}

We can now state and prove our main theorem.

THEOREM 2. A bipartite graph $G$ is Pfaffian if and only if, for every 1 -factor $f, G f$ does not contain a maximum odd ring.

REMARK. Clearly any ring in $G f$ is strict.

Proof. From Theorem 1 and Lemmas 1 and 2, it suffices to prove that a necessary and sufficient condition for the existence of a 1-factor $f$ such that $G^{*} f$ contains a maximum odd ring is the existence of a 1 -factor $f^{\prime}$ such that $G^{*} f^{\prime}$ contains a subdivision of $H$.

Suppose first that $f^{\prime}$ is a 1 -factor of $G$ such that $G^{*} f^{\prime}$ contains a subdivision of $H$. Then $G^{*} f^{\prime}$ contains a maximum odd ring by the argument at the beginning of the proof of the main theorem of [4].

Now let $f$ be a 1 -factor of $G$ such that $G^{*} f$ contains a maximum odd ring. Let $S$ be a maximum odd ring $\left\{C_{0}, \ldots, C_{n-1}\right\}$ in $G^{*} f$ such that $\left|\bigcup_{j=0}^{n-1} E\left(C_{j}\right)\right|$ is minimal. Without loss of generality, we assume that $G=\bigcup_{j=0}^{n-1} C_{j}$, for any edge not in a circuit of $S$ is irrelevant. It follows that for any maximum odd ring $S^{\prime}$ of $G^{*} f$, any edge of $G^{*} f$ belongs to some circuit of $S^{\prime}$. Furthermore, let $C_{0}, C_{1}, \ldots, C_{n-1}, C_{0}$ be the cyclic ordering of the elements of $S$ that satisfies condition (b) of the definition of a ring.

As each circuit of $S$ is a directed circuit, the argument now follows closely the proof of the main theorem of [4]. The condition that $G$ is planar is replaced by the condition that $G^{*} f$ contains a subdivision of $H$. If $G^{*} f$ does not contain a subdivision of $H$, then it can be shown as in [4], with only very minor modifications, that every $\bar{C}_{i} C_{i+1}$-chord contains edges in common with $C_{i+2}$ and every $\bar{C}_{i} C_{i-1}$-chord contains edges in common with $C_{i-2}$. The reorientations of edges that occur in the proof can be justified by appealing to Lemma 3 to show that they merely amount to choosing a new 1 -factor for $G$. In case $I A(2)$, the graph

$$
\bigcup_{j=0}^{k} P_{j} \cup \bigcup_{j=1}^{k-2} C_{i}\left(u_{j+2}, w_{j}\right) \cup C_{i}\left(w_{1}, w_{k-1}\right)
$$

becomes a subdivision of $H$ upon the reorientation of every edge of every circuit $P_{j}\left(u_{j}, w_{j}\right) C_{i}\left(w_{j}, u_{j}\right)$ for all odd $j$ such that $j>1$. 
As in [4], we now distinguish two cases, but the discussion of them is more involved.

Case I. Suppose $|S| \geqslant 5$. Since no edge belongs to more than two circuits of $S$, it follows that $C_{i} \neq C_{i+1}$ because $E\left(C_{i+1}\right) \cap E\left(C_{i+2}\right) \neq \varnothing$. Hence for any value of $i$ there must be a $\bar{C}_{i} C_{i+1}$-chord. We now introduce two subcases.

Case IA. Suppose that for some value of $i$ there exist a $\bar{C}_{i} C_{i+1}$-chord $P_{1}$ with origin $u$ and terminus $y$ and a $\bar{C}_{i} C_{i+1}$-chord $P_{2}$ with origin $w$ and terminus $x$, and that there vertices occur on $C_{i}$ in the cyclic order $x, v, w, u, x$. We have $E\left(P_{1}\right) \cap E\left(C_{i+2}\right) \neq \varnothing$ since $P_{1}$ is a $\bar{C}_{i} C_{i+1}$-chord. Let $y, z$ be two internal vertices of $P_{1}$ such that $P_{1}(y, z)$ is a $C_{i+1} C_{i+2}$-chord.

Suppose the choice of $y$ and $z$ is not unique. Clearly $z$ is determined if $y$ is given and vice versa (since $C_{i+1} C_{i+2}$-chords are by definition of maximal length); therefore let us suppose that there are $y^{\prime}, z^{\prime}$, where $\left\{y^{\prime}, z^{\prime}\right\} \cap\{y, z\}=\varnothing$, such that $P_{1}\left(y^{\prime}, z^{\prime}\right)$ is a $C_{i+1} C_{i+2}$-chord. By the definition of a $C_{i+1} C_{i+2}$-chord, these vertices must occur on $P_{1}$ in the order $y^{\prime}, z^{\prime}, y, z$ or $y, z, y^{\prime}, z^{\prime}$. Without loss of generality we choose the former order. Therefore $P_{1}\left(z^{\prime}, y\right)$ is a $\bar{C}_{i+2} C_{i+1}$-chord and hence must contain edges in common with $C_{i}$. It does not do so, however, because it is a subpath of a $\bar{C}_{i} C_{i+1}$-chord. This contradiction shows that the choice of $y$ and $z$ is unique.

Therefore $E\left(C_{i+2}\right) \cap E\left(P_{1}\right)=E\left[P_{1}(y, z)\right]$. Since $|S| \geqslant 5, E\left(C_{i+2}\right) \cap E\left(C_{i}\right)=$ $\varnothing$. However $E\left(C_{i+2}\right) \cap E\left(P_{2}\right) \neq \varnothing$ since $P_{2}$ is a $\bar{C}_{i} C_{i+1}$-chord. Therefore $P_{2}$ contains vertices $a, b$ such that $P_{2}(a, b)$ is a $C_{i+1} C_{i+2}$-chord. As before, $a$ and $b$ are chosen uniquely. In summary, paths $C_{i+2}(z, a)$ and $C_{i+2}(b, y)$ have no edges in common with $P_{1}, P_{2}$ or $C_{i}$. Hence the graph

$$
C_{i}(x, u) \cup C_{i+1}(w, a) \cup C_{i+1}(u, y) \cup C_{i+1}(z, v) \cup C_{i+1}(b, x) \cup C_{i+2}
$$

is a subdivision of $H$.

Case IB. The only other possibility is the case where, for every value of $i$ and every $\bar{C}_{i} C_{i+1}$-chord $P_{1}$ with origin $u_{1}$ and terminus $v_{1}$, there does not exist a $\bar{C}_{i} C_{i+1}$-chord with origin in $V\left[C_{i}\left(v_{1}, u_{1}\right)\right]$ and terminus in $V\left[C_{i}\left(u_{1}, v_{1}\right)\right]$. Clearly there is no $\bar{C}_{i} C_{i+1}$-chord with origin in $V\left[C_{i}\left(u_{1}, v_{1}\right)\right]$ either, for if there were, there would then have to be a $\bar{C}_{i} C_{i+1}$-chord with origin in $V\left[C_{i}\left(v_{1}, u_{1}\right)\right]$ and terminus in $V\left[C_{i}\left(u_{1}, v_{1}\right)\right]$, since $C_{i+1}$ is a circuit. This property will be referred to as the non-interlocking property of $\bar{C}_{i} C_{i+1}$-chords.

Without loss of generality, let $i=0$ so that $P_{1}$ is a $\bar{C}_{0} C_{1}$-chord. We have $E\left(P_{1}\right) \cap E\left(C_{2}\right) \neq \varnothing$, so that $P_{1}$ contains a $C_{1} C_{2}$-chord $P_{2}$ with origin $u_{2}$ and terminus $v_{2}$. As before, there is only one choice for $P_{2}$. There must be a $\bar{C}_{2} C_{3}$-chord since $C_{2} \neq C_{3}$. In particular, since no two $\bar{C}_{2} C_{3}$-chords can interlock, and since $E\left(C_{1}\right) \cap E\left(C_{3}\right)=\varnothing$, there must be a $\bar{C}_{2} C_{3}$-chord $P_{3}$ with origin $u_{3}$ and 
terminus $v_{3}$ in $C_{2}\left(v_{2}, u_{3}\right)$. Again since no two $\bar{C}_{2} C_{3}$-chords can interlock, the path $C_{2}\left(u_{3}, v_{3}\right)$ is a $\bar{C}_{3} C_{2}$-chord, and therefore contains a unique subpath, namely $P_{2}$, which is a $C_{1} C_{2}$-chord. Since $P_{3}$ is a $\bar{C}_{2} C_{3}$-chord, it contains a $C_{3} C_{4}$-chord $P_{4}$ with origin $u_{4}$ and terminus $v_{4}$. Since $C_{5} \neq C_{4}$, there must be a $\bar{C}_{4} C_{5}$-chord. Since no two $\bar{C}_{4} C_{5}$-paths can interlock, and since $E\left(C_{3}\right) \cap E\left(C_{5}\right)=\varnothing$, there must be a $\bar{C}_{4} C_{5}$-chord $P_{5}$ with origin $u_{5}$ and terminus $v_{5}$ in $C_{4}\left(v_{4}, u_{5}\right)$. We proceed in this manner until a path $P_{k}$ is found, where $k=|S|-1$. Since $k$ is even, $P_{k}$ must be a $C_{k-1} C_{k}$-chord.

Now we consider $C_{k}$. Let $u_{0}$ be the first vertex of the path $C_{k}\left(v_{k}, u_{k}\right)$ that belongs to $V\left(C_{0}\right)$. Let $v_{0}$ be the last vertex of $C_{k}\left(v_{k}, u_{k}\right)$ belonging to $V\left(C_{0}\right)$. Since $G^{*} f$ is cubic, we have $\left\{u_{0}, v_{0}\right\} \cap\left\{u_{1}, v_{1}\right\}=\varnothing$. There are now six subcases, corresponding to the six possible cyclic orderings of the vertices $u_{0}, v_{0}, u_{1}, v_{1}$ on $C_{0}$.

(1) Suppose that the vertices occur on $C_{0}$ in the cyclic order $v_{1}, u_{0}, u_{1}, v_{0}, v_{1}$. Clearly $u_{0}$ is the origin of a $C_{0} C_{k}$-chord. Such a chord cannot contain edges in common with $C_{1}$. Since the edge of $C_{0}$ with negative end $u_{1}$ and the edge of $C_{0}$ with positive end $v_{1}$ are both in $C_{1}$, it follows that there must exist a $\bar{C}_{0} C_{k}$-chord with origin $o$ lying on the path $C_{0}\left(v_{1}, u_{1}\right)$ and terminus $t$ lying on the path $C_{0}\left(u_{1}, v_{1}\right)$. The fact that no two $\bar{C}_{0} C_{k}$-chords can interlock implies that $o \in$ $V\left[C_{0}\left(u_{0}, u_{1}\right)\right]$ and $t \in V\left[C_{0}\left(u_{1}, v_{0}\right)\right]$.

Since $E\left(C_{1}\right) \cap E\left(C_{k}\right)=\varnothing$, there must be a $\bar{C}_{0} C_{1}$-chord with terminus $y \in$ $V\left[C_{0}\left(o, u_{1}\right)\right]$. Choose $y$ so that the length of the path $C_{0}(o, y)$ is minimal. This $\bar{C}_{0} C_{1}$-chord must have edges, and therefore vertices, in common with $C_{2}$; let $x$ be the last such vertex. Therefore $x$ is the terminus of a $C_{1} C_{2}$-chord; call the origin of this chord $x^{\prime}$. Since $C_{2}\left(u_{2}, v_{2}\right)$ is, as we have seen, the unique $C_{1} C_{2}$-chord which is a subpath of $C_{2}\left(u_{3}, v_{3}\right)$, it follows that $x$ and $x^{\prime}$ lie on $C_{2}\left(v_{3}, u_{3}\right)$. The vertex $x^{\prime}$ is the terminus of a $\bar{C}_{2} C_{1}$-chord, and this chord must have vertices in common with $C_{0}$; let $y^{\prime}$ be the last such vertex. Thus $C_{1}\left(y^{\prime}, y\right)$ is a $\bar{C}_{0} C_{1}$-chord.

Because no two $\bar{C}_{0} C_{1}$-chords can interlock, we have $y^{\prime} \in V\left[C_{0}\left(v_{1}, y\right)\right]$. Suppose $y^{\prime} \in V\left[C_{0}(o, y)\right]$. Then since $y^{\prime}$ is the terminus of a $C_{0} C_{1}$-chord, and since $E\left(C_{1}\right) \cap E\left(C_{k}\right)=\varnothing$, there must exist some vertex on the path $C_{0}\left(o, y^{\prime}\right)$ which is the terminus of a $\bar{C}_{0} C_{1}$-chord. This result contradicts the choice of $y$. Therefore $y^{\prime} \in V\left[C_{0}\left(v_{1}, o\right)\right]$. We distinguish two subcases.

(a) Suppose $y^{\prime} \in V\left[C_{0}\left(u_{0}, o\right)\right]$. (See Figure 2.)

Then $S^{\prime}=\left(C_{0}^{\prime}, C_{1}^{\prime}, \ldots, C_{n-1}^{\prime}\right)$ becomes an odd ring of directed circuits upon the reorientation of the edges of $C_{0}$, where $C_{j}^{\prime}=C_{j}$ for all even $j$ such that $2<j<k$, $C_{j}^{\prime}=C_{j}\left(u_{j}, v_{j}\right) C_{j-1}\left(v_{j}, u_{j}\right)$ for all odd $j$ such that $2<j<k, C_{0}^{\prime}=$ $C_{0}^{-1}\left(v_{1}, u_{1}\right) C_{1}\left(u_{1}, v_{1}\right), C_{1}^{\prime}=C_{0}^{-1}(t, o) C_{k}(o, t), C_{2}^{\prime}=C_{1}\left(y^{\prime}, y\right) C_{0}^{-1}\left(y, y^{\prime}\right)$ and $C_{k}^{\prime}$ $=C_{k}\left(v_{0}, u_{0}\right) C_{0}^{-1}\left(u_{0}, v_{0}\right)$. Furthermore $\left|S^{\prime}\right|=|S|$ and so $S^{\prime}$ is a maximum odd 


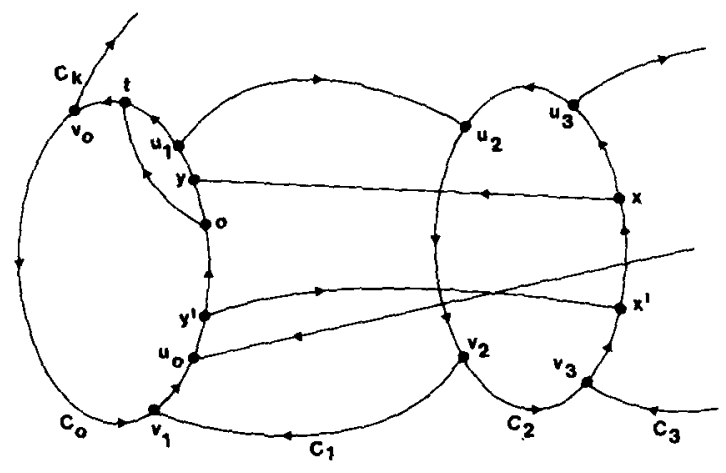

Figure 2

ring. However, there are edges of $G^{*} f$ that are not in any circuit of $S^{\prime}$. An example is the edge of $C_{2}$ with positive end $u_{3}$. The minimality property of $S$ is contradicted.

(b) Suppose $y^{\prime} \in V\left[C_{0}\left(v_{1}, u_{0}\right)\right]$. (See Figure 3.) Then $C_{0}\left(v_{1}, v_{0}\right) \cup C_{1}\left(u_{1}, u_{2}\right)$ $\cup C_{1}\left(v_{2}, v_{1}\right) \cup C_{1}(x, y) \cup C_{1}\left(y^{\prime}, x^{\prime}\right) \cup C_{2}\left(v_{3}, v_{2}\right) \cup \cup \cup_{j=2}^{k / 2} C_{2 j-1}\left(u_{2 j}, v_{2 j-1}\right)$ $\cup \cup_{j=2}^{(k-2) / 2} C_{2 j}\left(v_{2 j+1}, u_{2 j}\right) \cup C_{k}\left(v_{0}, u_{k}\right)$ is a subdivision of $H$.

(2) Suppose the vertices occur on $C_{0}$ in the cyclic order $v_{1}, u_{1}, v_{0}, u_{0}, v_{1}$. Clearly $C_{k}\left(v_{0}, u_{0}\right)$ is a $\bar{C}_{0} C_{k}$-chord; since no two such chords interlock, it follows that $C_{0}\left(v_{0}, u_{0}\right)$ is a $\bar{C}_{k} C_{0}$-chord. Therefore $E\left(C_{1}\right) \cap E\left[C_{0}\left(v_{0}, u_{0}\right)\right] \neq \varnothing$.

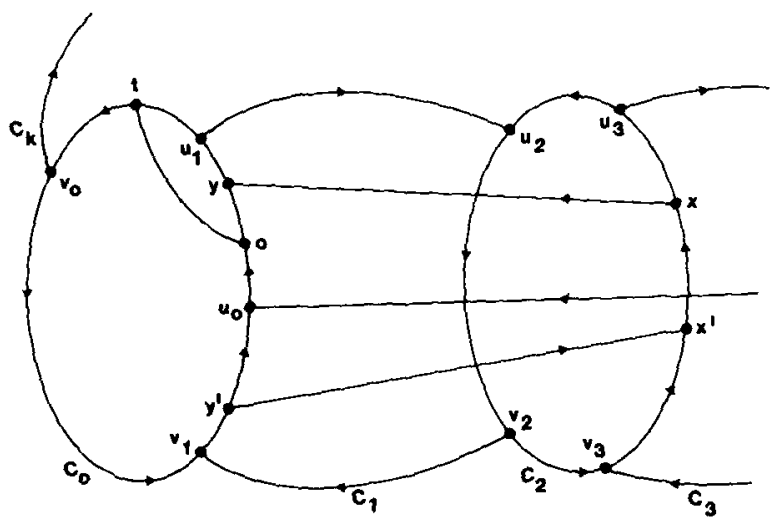

Figure 3 
Now we consider the $C_{0} C_{1}$-chords and the cyclic order in which they occur on $C_{0}$ and on $C_{1}$. One such chord has terminus $u_{1}$; it is succeeded on $C_{1}$, and therefore on $C_{0}$ also because of the prohibition of interlocking $\bar{C}_{0} C_{1}$-chords, by one whose origin is $v_{1}$. Hence there is no subpath of $C_{0}\left(u_{1}, v_{1}\right)$ which is a $C_{0} C_{1}$-chord. This fact contradicts the conclusion of the preceding paragraph.

(3) Suppose that the vertices occur on $C_{0}$ in the cyclic order $v_{1}, u_{1}, u_{0}, v_{0}, v_{1}$. Then a subdivision of $H$ can be produced after a suitable reorientation of edges.

The remaining possibilities reduce to the ones already considered after reorientation of the edges of $C_{0}$. The case where $|S| \geqslant 5$ is now complete.

Case II. Suppose $|S|=3$. Let $S=\left\{C_{0}, C_{1}, C_{2}\right\}$. First we consider $C_{0}$ and $C_{1}$.

Case IIA. Suppose there exist a $\bar{C}_{0} C_{1}$-chord with origin $o_{1}$ and terminus $t_{1}$ and another $\bar{C}_{0} C_{1}$-chord with terminus $t \in V\left[C_{0}\left(o_{1}, t_{1}\right)\right]$.

Since $C_{1}$ is a directed circuit, it is then obvious that there must be a $\bar{C}_{0} C_{1}$-chord with origin $o_{2} \in V\left[C_{0}\left(t_{1}, o_{1}\right)\right]$ and terminus $t_{2} \in V\left[C_{0}\left(o_{1}, t_{1}\right)\right]$, for otherwise every edge of $E\left(C_{1}\right) \cap E\left(C_{0}\right)$ would be on the path $C_{0}\left(t_{1}, o_{1}\right)$ and this result would contradict the existence of $t$. There may be many choices for this $\bar{C}_{0} C_{1}$ chord; we choose the one such that the length of $C_{1}\left(o_{1}, t_{2}\right)$ is minimal. Since $C_{1}$ is a directed circuit, it is again clear that some subpath of $C_{1}\left(t_{2}, o_{1}\right)$ is a $\bar{C}_{0} C_{1}$-chord with origin $o_{3} \in V\left[C_{0}\left(o_{1}, t_{1}\right)\right]$ and terminus $t_{3} \in V\left[C_{0}\left(t_{1}, o_{1}\right)\right]$. We choose this path so that the length of $C_{1}\left(t_{2}, o_{3}\right)$ is minimal. There now arise several cases and subcases.

(1) Suppose $o_{3} \in V\left[C_{0}\left(o_{1}, t_{2}\right)\right]$.

(a) Suppose $t_{3} \in V\left[C_{0}\left(t_{1}, o_{2}\right)\right]$.

(i) Suppose there exists a $\bar{C}_{0} C_{1}$-chord with origin $o_{4} \in V\left[C_{0}\left(t_{2}, t_{1}\right)\right]$ and terminus $t_{4} \in V\left[C_{0}\left(o_{1}, o_{3}\right)\right]$.

Define the circuits

$C_{0}^{\prime}=C_{0}\left(t_{4}, o_{4}\right) C_{1}\left(o_{4}, t_{4}\right)$,

$C_{1}^{\prime}=C_{0}\left(t_{3}, o_{3}\right) C_{1}\left(o_{3}, t_{3}\right)$

$C_{2}^{\prime}=C_{0}\left(t_{2}, o_{2}\right) C_{1}\left(o_{2}, t_{2}\right)$

Let $S^{\prime}=\left\{C_{0}^{\prime}, C_{1}^{\prime}, C_{2}^{\prime}\right\}$. Then $S^{\prime}$ is a maximum odd ring of directed circuits. However, no edge of the path $C_{1}\left(o_{1}, t_{1}\right)$ belongs to any circuit of $S^{\prime}$. This result contradicts the minimality property of $S$.

(ii) Suppose there is no $\bar{C}_{0} C_{1}$-chord with origin lying on $C_{0}\left(t_{2}, t_{1}\right)$ and terminus lying on $C_{0}\left(o_{1}, o_{3}\right)$. Since $C_{1}$ is a directed circuit, there must be a $\bar{C}_{0} C_{1}$-chord with origin $u_{1} \in V\left[C_{0}\left(t_{2}, t_{1}\right)\right]$. Let $v_{1}$ be the terminus of this path. Since $u_{1} \in$ $V\left[C_{0}\left(t_{2}, t_{1}\right)\right], u_{1} \neq o_{3}$ and therefore if we choose $u_{1}$ so that $u_{1} \in V\left[C_{1}\left(t_{2}, o_{3}\right)\right]$, then, by the choice of $o_{3}, v_{1}$ cannot lie on the path $C_{0}\left(t_{1}, o_{1}\right)$. By the assumption defining case (ii), $v_{1}$ cannot lie on the path $C_{0}\left(o_{1}, o_{3}\right)$. If we choose $u_{1}$ to be the 
last vertex of the path $C_{1}\left(t_{2}, o_{3}\right)$ that lies on the path $C_{0}\left(t_{2}, t_{1}\right)$, then $v_{1}$ cannot lie on the path $C_{0}\left(t_{2}, t_{1}\right)$ either. Hence $v_{1} \in V\left[C_{0}\left(o_{3}, t_{2}\right)\right]$.

Again since $C_{1}$ is a directed circuit, there must be a subpath of $C_{1}\left(v_{1}, o_{3}\right)$ which is a $\bar{C}_{0} C_{1}$-chord with origin $u_{2} \in V\left[C_{0}\left(v_{1}, t_{2}\right)\right]$. Again we can choose $u_{2}$ to be the last vertex of $C_{1}\left(t_{2}, o_{3}\right)$ lying on $C_{0}\left(v_{1}, t_{2}\right)$. If the terminus of this $\bar{C}_{0} C_{1}$-chord is $v_{2}$, then as before $v_{2} \in V\left[C_{0}\left(o_{1}, v_{1}\right)\right]$. Suppose $v_{2} \in V\left[C_{0}\left(o_{3}, v_{1}\right)\right]$. Then, since $C_{1}$ is a directed circuit, there must be a subpath of $C_{1}\left(v_{2}, o_{3}\right)$ which is a $\bar{C}_{0} C_{1}$-chord with origin $u_{3} \in V\left[C_{0}\left(v_{2}, v_{1}\right)\right]$. Let the terminus of this path be $v_{3}$. We choose $u_{3}$ again so that it is the last vertex of $C_{1}\left(t_{2}, o_{3}\right)$ lying on the path $C_{0}\left(v_{2}, v_{1}\right)$. Then $v_{3} \in V\left[C_{0}\left(o_{1}, v_{2}\right)\right]$. If $v_{3} \in V\left[C_{0}\left(o_{3}, v_{2}\right)\right]$, then we repeat the argument. By the finiteness of the graph, there must exist an integer $n \geqslant 2$ such that $v_{n} \in$ $V\left[C_{0}\left(o_{1}, o_{3}\right)\right]$.

Define the following circuits:

$$
\begin{aligned}
& C_{j}^{\prime}=C_{0}\left(v_{j}, u_{j}\right) C_{1}\left(u_{j}, v_{j}\right) \text { for all } j \in\{1,2, \ldots, n\}, \\
& C_{n+1}^{\prime}=C_{0}\left(t_{3}, o_{3}\right) C_{1}\left(o_{3}, t_{3}\right), \\
& C_{n+2}^{\prime}=C_{0}\left(t_{2}, o_{2}\right) C_{1}\left(o_{2}, t_{2}\right) .
\end{aligned}
$$

Let $S^{\prime}=\left\{C_{1}^{\prime}, C_{2}^{\prime}, \ldots, C_{n+2}^{\prime}\right\}$. Then $\left|S^{\prime}\right|=n+2 \geqslant 4$. Now $S^{\prime}$ is clearly an odd ring of directed circuits, and so the maximality of $S$ is contradicted.

(b) Suppose $t_{3} \in V\left[C_{0}\left(o_{2}, o_{1}\right)\right]$. (This is the only other possibility since by assumption $t_{3} \in V\left[C_{0}\left(t_{1}, o_{1}\right)\right]$.) In this case, we define vertices $u_{i}$ and $v_{i}$ as in Case (a)(ii) for all $i \in\{1,2, \ldots, n\}$. Again $v_{n} \in V\left[C_{0}\left(o_{1}, o_{3}\right)\right]$ but in this case $n \geqslant 1$. Now define the following circuits:

$$
\begin{aligned}
& C_{j}^{\prime}=C_{0}\left(v_{j}, u_{j}\right) C_{1}\left(u_{j}, v_{j}\right) \text { for all } j \in\{1,2, \ldots, n\}, \\
& C_{n+1}^{\prime}=C_{0}\left(t_{3}, o_{3}\right) C_{1}\left(o_{3}, t_{3}\right), \\
& C_{n+2}^{\prime}=C_{0}\left(t_{1}, o_{1}\right) C_{1}\left(o_{1}, t_{1}\right), \\
& C_{n+3}^{\prime}=C_{0}\left(t_{2}, o_{2}\right) C_{1}\left(o_{2}, t_{2}\right) .
\end{aligned}
$$

Let $S^{\prime}=\left\{C_{1}^{\prime}, C_{2}^{\prime}, \ldots, C_{n+3}^{\prime}\right\}$. Then $\left|S^{\prime}\right|=n+3 \geqslant 4$. Again the maximality of $S$ is contradicted.

(2) Suppose $o_{3} \in V\left[C_{0}\left(t_{2}, t_{1}\right)\right]$. There are two subcases.

(a) Suppose $t_{3} \in V\left[C_{0}\left(o_{2}, o_{1}\right)\right]$. Then $C_{0} \cup C_{1}\left(o_{1}, t_{1}\right) \cup C_{1}\left(o_{2}, t_{2}\right) \cup C_{1}\left(o_{3}, t_{3}\right)$ is a subdivision of $\boldsymbol{H}$.

(b) The only other possibility is the case where $t_{3} \in V\left[C_{0}\left(t_{1}, o_{2}\right)\right]$.

Define $u_{1}=o_{2}, v_{1}=t_{2}, u_{2}=o_{1}, v_{2}=t_{1}, u_{3}=o_{3}, v_{3}=t_{3}$. Since we have disposed of all the other possibilities we are free to assume that all $\bar{C}_{0} C_{1}$-chords considered in Case A satisfy Case (2)(b).

Since $C_{1}$ is a circuit, there must clearly be a $\bar{C}_{0} C_{1}$-chord with origin $u_{4} \in$ $V\left[C_{0}\left(u_{3}, v_{3}\right)\right]$ and terminus $v_{4} \in V\left[C_{0}\left(v_{3}, u_{3}\right)\right]$. We choose $u_{4}$ so that the length of the path $C_{1}\left(u_{2}, u_{4}\right)$ is minimal. Furthermore $u_{4} \in V\left[C_{0}\left(v_{2}, v_{3}\right)\right]$, because if 
$u_{4} \in V\left[C_{0}\left(u_{3}, v_{2}\right)\right]$, then the $\bar{C}_{0} C_{1}$-chords $C_{1}\left(u_{3}, v_{3}\right), C_{1}\left(u_{2}, v_{2}\right)$ and $C_{1}\left(u_{4}, v_{4}\right)$ together would satisfy Case (1).

Suppose $v_{4} \in V\left[C_{0}\left(v_{3}, u_{1}\right)\right]$. Then since $C_{1}$ is a circuit, there must be a $\bar{C}_{0} C_{1}$-chord with origin $u_{5} \in V\left[C_{0}\left(u_{4}, v_{4}\right)\right]$ and terminus lying on the path $C_{0}\left(v_{4}, u_{4}\right)$. Again, choose $u_{5}$ in such a way that the length of the path $C_{1}\left(u_{3}, u_{5}\right)$ is minimal. We have $u_{5} \in V\left[C_{0}\left(v_{3}, v_{4}\right)\right]$ because if $u_{5} \in V\left[C_{0}\left(u_{4}, v_{3}\right)\right]$ then the $\bar{C}_{0} C_{1}$-chords $C_{1}\left(u_{4}, v_{4}\right), C_{1}\left(u_{3}, v_{3}\right)$ and $C_{1}\left(u_{5}, v_{5}\right)$ together would satisfy Case (1).

If $v_{5} \in V\left[C_{0}\left(v_{4}, u_{1}\right)\right]$, we repeat the argument. By the finiteness of the graph, there is an integer $n$ such that $v_{n} \notin V\left[C_{0}\left(v_{n-1}, u_{1}\right)\right]$. Therefore there must exist an integer $m$ such that $v_{n} \in V\left[C_{0}\left(u_{m}, u_{m+1}\right)\right]$. Since by definition $v_{n} \notin$ $V\left[C_{0}\left(u_{n-1}, u_{n}\right)\right]$, we must have $m \leqslant n-2$.

(i) Suppose $m=n-2$. Then $C_{0} \cup C_{1}\left(u_{n-2}, v_{n-2}\right) \cup C_{1}\left(u_{n-1}, v_{n-1}\right) \cup$ $C_{1}\left(u_{n}, v_{n}\right)$ is a subdivision of $H$.

(ii) Suppose $m<n-2$. Then the set

$$
\begin{array}{r}
S^{\prime}=\left\{C_{0}^{-1}\left(v_{m}, u_{m}\right) C_{1}\left(u_{m}, v_{m}\right), C_{0}^{-1}\left(v_{m+1}, u_{m+1}\right) C_{1}\left(u_{m+1}, v_{m+1}\right)\right. \\
\left.\ldots, C_{0}^{-1}\left(v_{n}, u_{n}\right) C_{1}\left(u_{n}, v_{n}\right)\right\}
\end{array}
$$

becomes an odd ring of directed circuits upon reorientation of every edge of $C_{0}$. Since $m \leqslant n-3$, we have $\left|S^{\prime}\right|=n-m+1 \geqslant 4$, so that the maximality of $|S|$ is contradicted.

Case IIB. We now assume that for every $\bar{C}_{0} C_{1}$-chord with origin $o_{1}$ and terminus $t_{1}$ there does not exist a $\bar{C}_{0} C_{1}$-chord whose terminus lies on the path $C_{0}\left(o_{1}, t_{1}\right)$. It obviously follows that there is no $\bar{C}_{0} C_{1}$-chord with origin in $V\left[C_{0}\left(o_{1}, t_{1}\right)\right]$. Thus no two $\bar{C}_{0} C_{1}$-chords interlock. We can clearly assume also that no two $\bar{C}_{1} C_{0^{-}}, \bar{C}_{2} C_{1^{-}}, \bar{C}_{1} C_{2}-, \bar{C}_{0} C_{2^{-}}$or $\bar{C}_{2} C_{0^{-}}$-chords interlock either, for otherwise we simply apply Case $A$ to the appropriate pair of circuits.

We recall that every $\bar{C}_{0} C_{1}$-chord contains edges in common with $C_{2}$. Of course, corresponding statements hold for $\bar{C}_{1} C_{0^{-}}, \bar{C}_{0} C_{2^{-}}, \bar{C}_{2} C_{0^{-}}, \bar{C}_{2} C_{1^{-}}$and $\bar{C}_{1} C_{2}$-chords.

Suppose there are distinct $\bar{C}_{0} C_{1}$-chords $P_{1}$ and $P_{2}$. Let $P_{1}$ have origin $u_{1}$ and terminus $v_{1}$. Since $P_{1}, P_{2}$ are both $\bar{C}_{0} C_{1}$-chords, each of them contains edges in common with $C_{2}$. Hence some subpath of $P_{1}$ is a $C_{1} C_{2}$-chord; let this chord have origin $y_{1}$ and terminus $z_{1}$. Similarly, since $C_{0}\left(u_{1}, v_{1}\right)$ is a $\bar{C}_{1} C_{0}$-chord, it contains edges in common with $C_{2}$. Hence some subpath of $C_{0}\left(u_{1}, v_{1}\right)$ is a $C_{0} C_{2}$-chord; let this subpath have origin $w_{1}$ and terminus $x_{1}$.

Define the following circuits:

$$
C_{0}^{\prime}=C_{0}\left(v_{1}, u_{1}\right) C_{1}\left(u_{1}, v_{1}\right), \quad C_{1}^{\prime}=C_{1}\left(v_{1}, u_{1}\right) C_{0}\left(u_{1}, v_{1}\right) .
$$

Let $S^{\prime}=\left\{C_{0}^{\prime}, C_{1}^{\prime}, C_{2}\right\}$. $S^{\prime}$ is clearly a maximum odd ring of directed circuits.

We now consider the set of $C_{0}^{\prime} C_{2}$ - and $C_{1}^{\prime} C_{2}$-chords, and the cyclic order in which they occur on the directed circuit $C_{2} . C_{0}\left(w_{1}, x_{1}\right)$ is an example of a 
$C_{1}^{\prime} C_{2}$-chord. Since $P_{2}$ is a $\bar{C}_{0}^{\prime} C_{1}^{\prime}$-chord, some subpath of $P_{2}$ must be another $C_{1}^{\prime} C_{2}$-chord distinct from $C_{0}\left(w_{1}, x_{1}\right)$. Therefore the $C_{1}^{\prime} C_{2}$-chord $C_{0}\left(w_{1}, x_{1}\right)$ cannot be both preceded and followed on $C_{2}$ by the $C_{0}^{\prime} C_{2}$-chord $C_{1}\left(y_{1}, z_{1}\right)$ without any intervening $C_{0}^{\prime} C_{2^{-}}$or $C_{1}^{\prime} C_{2}$-chords. For the sake of concreteness, suppose that $C_{0}\left(w_{1}, x_{1}\right)$ is followed on $C_{2}$ by $P_{3}$ without any intervening $C_{0}^{\prime} C_{2}$ - or $C_{1}^{\prime} C_{2}$-chords, where $P_{3} \neq C_{0}^{\prime}\left(y_{1}, z_{1}\right)$ and $P_{3}$ is either a $C_{0}^{\prime} C_{2^{-}}$or a $C_{1}^{\prime} C_{2}$-chord. Suppose the former. Then $P_{3}$ cannot be a subpath of $C_{1}\left(u_{1}, v_{1}\right)$ because $C_{1}\left(y_{1}, z_{1}\right)$ is the unique $C_{1} C_{2}$-chord which is a subpath of $C_{1}\left(u_{1}, v_{1}\right)$. Thus if $P_{3}$ is a $C_{0}^{\prime} C_{2}$-chord, it is clearly a $C_{0} C_{2}$-chord. If $P_{3}$ has origin $o$, then if follows from the definition of $P_{3}$ that $C_{2}\left(x_{1}, o\right)$ is a $\bar{C}_{0} C_{2}$-chord that contains no edges in common with $C_{1}$. This is a contradiction; hence $P_{3}$ must be a $C_{1}^{\prime} C_{2}$-chord. But now the path $C_{2}\left(x_{1}, o\right)$ is a $\bar{C}_{1} C_{2}$-chord containing no edges in common with $C_{0}^{\prime}$, contradicting the fact that $S^{\prime}$ is a maximum odd ring. Thus we have a contradiction in either case. The argument is similar if $C_{0}\left(w_{1}, x_{1}\right)$ is not preceded on $C_{2}$ by $C_{1}\left(y_{1}, z_{1}\right)$ without any intervening $C_{0}^{\prime} C_{2}$ - or $C_{1}^{\prime} C_{2}$-chords.

We conclude that there is exactly one $\bar{C}_{0} C_{1}$-chord, $P$, say. Let $P$ have origin $u$ and terminus $v$. Then $C_{2}$ must contain a $C_{1} C_{2}$-chord which is a subpath of $P$. Let this chord have origin $y$ and terminus $z$. Similarly $C_{2}$ must contain a $C_{0} C_{2}$-chord which is a subpath of $C_{0}(u, v)$; let this chord have origin $w$ and terminus $x$. Then $C_{2}(x, y)$ and $C_{2}(z, w)$ have no internal vertices in common with $C_{1}$ or $C_{2}$. Therefore the graph $C_{0} \cup C_{1} \cup C_{2}$ is a subdivision of $H$. The theorem is proved.

\section{References}

[1] A. A. Chernyak, 'On an hypothesis of Little on planar graphs', (Russian) Izv. Akad. Nauk BSSR Ser. Fiz. Mat. No. 2 (1980), 41-45.

[2] P. W. Kasteleyn, 'Graph theory and crystal physics,' pp. 43-110, Graph theory and theoretical physics, (Academic Press, New York, 1967).

[3] C. H. C. Little, 'A characterization of convertible $(0,1)$-matrices', J. Comb. Theory Ser. B 18 (1975), 187-208.

[4] C. H. C. Little, 'A characterization of planar cubic graphs', J. Comb. Theory Ser. B 29 (1980), 185-194.

Department of Mathematics and Statistics

Massey University

Palmerston North

New Zealand 